\title{
Classical statistical entropy of black hole
}

\author{
Dipo Mahto', Ved Prakash ${ }^{2}$, Krishna Murari Singh ${ }^{1}$, Brajnandan Kumar ${ }^{2}$ \\ ${ }^{1}$ Department of Physics, Marwari College, T. M. B. U. Bhagalpur, Bhagalpur, India \\ ${ }^{2}$ University Department of Statistics \& Computer Application, T. M. B. U. Bhagalpur, Bhagalpur, India
}

Email address:

dipomahto@hotmail.com (D. Mahto), chandanbgp@gmail.com (V. Prakash),kmsinghphy@gmail.com (K. M. Singh), sonubgpkh@gmail.com (B. Kumar)

\section{To cite this article:}

Dipo Mahto, Ved Prakash, Krishna Murari Singh, Brajnandan Kumar. Classical Statistical Entropy of Black Hole. American Journal of Theoretical and Applied Statistics. Special Issue: Computational Statistics. Vol. 4, No. 1-1, 2015, pp. 15-18.

doi: 10.11648/j.ajtas.s.2015040101.13

\begin{abstract}
The present article derives an expression for classical statistical entropy of black hole using Maxwell- Boltzmann statistics and shows that the classical statistical entropy is directly proportional to the area of event horizon of black hole leading the result as $S_{b h} \alpha A(r)$. No primary and secondary data is used in this paper. We have designed the work similar to the work of Ren Zhao and Shuang-Qi Hu who obtained the quantum statistical entropy corresponding to the black hole horizon using Femi-Dirac $\&$ Bose-Einstein statistics. They have also shown that the entropy corresponding to the black hole horizon surface is the entropy of quantum state near the surface of the horizon. It is completely theoretical based work using Laptop done at Marwari College research laboratory and the residential research chamber of first author.
\end{abstract}

Keywords: Statistical Entropy, Event Horizon, Black Hole

\section{Introduction}

In the thermodynamical theory, the entropy is defined as the thermal properties of the substance which remains constant during a adiabatic change and a function of the state. It does not depend on a particular reversible process by which the substance is brought from and state to another state[1]. In fact the entropy is a measure of degree of randomness on the assembly. The entropy of a physical assembly in a definite state depends solely on the probability of that state. A relation between the entropy and probability of a state was established by Maxwell-Boltzmann by equation

$$
S=k \log \Omega
$$

where k is Boltzmann constant and $\Omega$ be the probability[2]. From 1904, the formula $S=k \log \Omega$ was repeatedly criticized by Einstein. Einstein argued that one cannot possibly obtain $a$ priori the probabilities to find the dynamical system in a certain region in its phase space since these probabilities $(\Omega)$ must follow a posteriori from dynamics of the system[3].

The entropy of a black Hole is one of the important subjects in the theoretical physics [4]. In the general relativity of Einstein, the black hole's entropy is a simple geometrical quantity [5]. The pioneering work in which Hawking interpreted the quantum effect of black holes as the fact the event horizon emits thermal radiation spectral particles sets an epochal milestones for black hole physics[4,6].

On the other hand, Bekenstein and Hawking proposed that the entropy of block hole is proportional to the area of the horizon $[6,7,8]$. The brick-wall model advanced by Hooft gives a statistical method for calculating the black hole entropy by taking the entropy of the quantum state of the black hole[9]. Ren Zhao and Shuang Qi Hu derived the Bekenstein Hawking entropy for each static state black hole using Hooft's model and found that the quantum state density is divergent was the black hole horizon and entropy is mostly due to the contribution of the quantum state near the horizon surface [10]. Using the generalized uncertainty derived the quantum statistical entropy of Bosonic \& Fermionic field of a black hole and shown that it is proportional to the area of the horizon[11]. Zhao Ren et al. considered the corrections to all orders in Planck length on the quantum state density from a generalized uncertainty principle (GUP) and calculated the statistical entropy of the Bose field and Fermi field on the background of the four-dimensional spherically symmetric black holes without any cut off to show that the statistical entropy is directly proportional to the area of event horizon[12].

In the present research work, we have shown that the 
classical statistical entropy of black hole is directly proportional to the area of the event horizon of black hole on the basis of Maxwell-Boltzmann statistics as the quantum statistical entropy of the Bose field and Fermi field of black hole is directly proportional to the area of event horizon (R. Zhao et al. 2006, R. Zhao and Shuang Qi Hu, 2006, R. Zhao et al. 2008).

\section{Theoretical Model of Black Hole}

The first statistical derivation of the black hole entropy formula was suggested by Strominger on the basis of the conformal field theory (CFT) [13].

R. Zhao et al. obtained that the entropy of Bose system is not only proportional to the area of horizon, but also the entropy of Fermi system is proportional to the area of horizon [11] and the quantum statistical entropy of black hole using Bose Einstein Statistics is given by [11].

$$
\begin{gathered}
S_{b}=j \frac{V_{3}}{16(2 \pi)^{4}} \frac{A\left(r_{h}\right)}{f^{\frac{1}{6}}\left(r_{h}\right) \lambda^{2}}\left[\frac{1}{3} \sqrt{\frac{2 \varepsilon}{\kappa}+\frac{\pi}{8} \beta_{h} \frac{\varepsilon}{f^{\frac{1}{2}}\left(r_{h}\right) \lambda^{\frac{1}{2}}}}\right] \\
S_{b}=\alpha A\left(r_{h}\right)
\end{gathered}
$$

The above equation shows that the quantum statistical entropy of black hole is proportional to the horizon area. where

$$
\alpha=j \frac{V_{3}}{16(2 \pi)^{4}} \frac{1}{\lambda^{3 / 2}}\left[\frac{2}{3}+\frac{\pi^{2}}{2}\right]
$$

And the term $A\left(r_{h}\right)$ is the surface area of the black hole.

The quantum statistical entropy of black hole using Fermi-Dirac Statistics is given by [11].

$$
\begin{gathered}
S_{h}=i \frac{V_{3} \beta_{0}^{2}}{48(2 \pi)^{4}} \int \frac{A(r) \sqrt{g_{r r}(r)}-\left(g_{t t}(r)\right) d r}{\lambda^{3}}=i \frac{V_{3}}{72 \pi^{2} \lambda^{3 / 2}} A\left(r_{h}\right) \\
\text { Or } S_{h}=i \frac{V_{3}}{72 \pi^{2} \lambda^{3 / 2}} A\left(r_{h}\right)
\end{gathered}
$$

Hence, we can write the above equation as:

$$
S_{h} \alpha A(r)
$$

where

$$
i \frac{V_{3}}{72 \pi^{2} \lambda^{3 / 2}}=\text { Cons } \tan t
$$

where $\mathrm{i}$ is the degeneracy of the Fermion.

The equation (7) shows that the entropy is proportional to the horizon area of black hole.

Now let us define the partition function as: The sum over states is called the partition function and represented by capital letter Z. In principle, it is a sum over all the particle states of a system, and therefore contains the statistical information about the system. All of the thermodynamic properties of the system are derivable from the partition function. For a Maxwell-Boltzmann statistical system, the partition function is given by[2].

$$
Z=\sum_{i} g_{i} e^{-\frac{\varepsilon_{i}}{k T}}
$$

where $\mathrm{k}$ is Boltzmann constant and assuming that

$$
\beta=\frac{1}{k T}
$$

This constant beta has a significant physical meaning and inversely proportional to the temperature.

Taking logarithm on both sides, we have

$$
\ln Z=\sum_{i} g_{i} \ln \left(e^{-\beta \varepsilon_{i}}\right)
$$

For five-dimensional space-time, the area of a three-dimensional curved surface at any arbitrary point $r$ is given by[11].

$$
A(r)=r^{3} V_{3}
$$

Where $V_{3}$ is the volume of a unit three dimensional hyper-surface. Thus partition function of the system at the lamella with arbitrary thickness at a point outside the horizon is

$$
\ln Z=j \int A(r) \sqrt{g_{r r}(r)} d r \sum_{i} g_{i} \ln \left(e^{-\beta \varepsilon_{i}}\right)
$$

The free energy of the system is given by [4].

$$
\begin{gathered}
F=-\frac{1}{\beta_{0}} \ln Z \\
F=-\frac{j \int A(r) \sqrt{g_{r r}(r) d r} \sum_{i} \ln \left(e^{-\beta \varepsilon_{i}}\right)}{\beta_{0}}
\end{gathered}
$$

The entropy of the system was the black hole event horizon is given by[4].

$$
S_{b h}=\beta^{2} \frac{\partial F}{\partial \beta_{0}}
$$

Where

$$
\beta=\beta_{0} \sqrt{-g_{t t}(r)}
$$

Putting the value of $\mathrm{F}$ from equation (15) and $\beta$ from equation (17) in the equation (16), we have

$$
\begin{gathered}
S_{b h}=-j \beta_{0} \frac{\partial}{\partial \beta_{0}}\left[\int A(r) \sqrt{g_{r r}(r)} d r \sum_{i} \ln \left(e^{-\beta \varepsilon_{i}}\right)\right] \\
S_{b h}=-j \beta_{0} \frac{\partial}{\partial \beta_{0}}\left[\int A(r) \sqrt{g_{r r}(r)} d r \sum_{i} \ln \left(e^{-\beta_{0} \sqrt{g_{t t}(r)} \varepsilon_{i}}\right)\right]
\end{gathered}
$$


After the solution of above equation, we may show that

$$
S_{b h} \alpha A(r)
$$

The equation (20) shows that the classical statistical entropy of black hole is directly proportional to the area of the event horizon of black hole.

\section{Supporting Facts between the Area and Entropy of Black Holes}

Stephen Hawking showed under general conditions that the total area of classical black holes can never decrease, even if they collide and merge[14] which means that $\delta A \geq 0$ (second law of black hole mechanics).

Bekenstein suggested that a physical identification does hold between the laws of thermodynamics and the laws of black hole mechanics[15] and proposed that a black hole should have an entropy, and that it should be proportional to its horizon area[16] leading that $\delta S \geq 0$.

\section{Applicability of the Model}

This theoretical model of black hole can be applied to calculate the entropy of system of black holes consisting of classical perfect gas, ideal gas, one dimensional harmonic oscillator, monatomic and diatomic gas molecules.

\section{Results and Discussion}

Maxwell-Boltzmann Statistics is the classical case applicable for the distinguishable particles and any number of particles can be in any state.

Bose-Einstein Statistics is a quantum mechanical case and is applicable for indistinguishable particles. These particles are called bosons having integer spin: $0,1,2$, etc. and any number of particles can be in one state.

Fermi-Dirac Statistics is also quantum mechanical case and is applicable for indistinguishable particles. These particles are known as fermions having half integer spin: $1 / 2,3 / 2$, etc. In this case one particle can be in any one single particle state.

Ren Zhao and Shuang-Qi Hu introduced the generalized uncertainty relation to study the quantum statistical entropy of a black hole in higher- dimensional space time and derived quantum statistical entropy of Bosonic \&Fermionic field proportional to the area of the horizon of the black hole. This shows that the quantum treatment regarding the entropy is correct.

In the present work, we have used the partition function due to Maxwell-Boltzmann distribution law and showed that the classical statistical entropy of black hole is directly proportional to the area of the event horizon of black hole as the quantum statistical entropy of the Bose field and Fermi field of black hole is directly proportional to the area of event horizon (R. Zhao et al. 2006, R. Zhao and Shuang Qi Hu, 2006, R. Zhao et al. 2008).

\section{Conclusion}

During the study of present research work, we can draw the following conclusions such as:

1. The classical statistical entropy of black hole is directly proportional to the area of event horizon when the system of particles of black holes obeying Maxwell-Boltzmann statistics.

2. The black hole entropy is proportional to its horizon area leading that $\delta S \geq 0$.

\section{Acknowledgements}

Authors acknowledge their gratitude to the referee for pointing out the errors in original manuscript and making constructive suggestions.

\section{References}

[1] Gupta S. L. \& Kumar V. "Elementary Statistical Mechanics." Pragati Prakashan, Meerut, ISBN-81-7556-988-3(2007).

[2] Alfred,H.www.scribd.com/doc/22056940/statisticalmechanics (2009).

[3] Cohen, E. G. D. "Boltzmann and Einstein: Statistics and dynamics-An unsolved problem." Pramana 64.5 (2005): 635-643.

[4] Zhao R., Zhang Z. and Zhang S. "Uncertainty relation and black hole entropy of NUT-Kerr Newmann space-time." Commun. Theoretical Physics (Beijing, China), Doi 10.393/ncb/i2004-10191-9, Vol.120B.1(2005):61-67.

[5] Zhao R., Zhang J. and Zhang L. "Statistical entropy of axial symmetry Einstein-Maxwell-Dilation axion black holes." Bulgarian Journal of Physics 28.5/6(2001): 200-208.

[6] Hawking S. W. "Particle creation by black hole." Communication in Mathematical Physics. 43(1975):199-220.

[7] Bekenstein J. D. "Black holes and Entropy." Phys. Rev. D 7, (1973):2333-2346.

[8] G. W. Gibbons and S. W. Hawking: Cosmological event horizons, thermodynamics and particle creation, Phys. Rev. D 15, (1977): 2738.

[9] Hooft G.'t “'On quantum structure of a black hole.” Nucl. Phys. B256, (1985):727-745.

[10] Zhao R. and Shuang-Qi H. "Quantum Statistical Entropy of the 5-Dimensional Stringy Black Hole." Chinese Journal of Physics, 44.3(2006):172-179.

[11] Zhao R., Wu Y. and Zhang S.(2006): "Quantum Statistical Entropy of Five-Dimensional Black Hole." Commun. Theoretical Physics(Beijing, China) 45.5(2006):849-852.

[12] Zhao R., Zhang L., Wu Y., and Li H.(2008): "Entropy of Four-Dimensional Spherically Symmetric Black Holes with Planck Length." Commun. Theoretical Physics (Beijing, China) 50.6(2008):1327-1330.

[13] Strominger, A. "Black hole entropy from near horizon microstates.” J. High Energy Phys. 02(1998):1-10. 
[14] Hawking, S. "A Brief History of Time.” Bantam Books, New York. ISBN:0-553-38016-8(1998).

[15] Transchen, J. "An introduction to black hole evaporation." arXiv: gr-qc/0010055V1(2000):1-32.
[16] Wald, R.M. "The thermodynamics of black hole." Living Reviews in Relativity, 4(2001):1-42. 